\title{
Some Lacunary Sequence Spaces of Invariant Means Defined by Musielak-Orlicz Functions on 2-Norm Space
}

\author{
Mohammad Aiyub \\ Department of Mathematics, University of Bahrain, Sakhir, Bahrain \\ Email: maiyub2002@gmail.com
}

Received 11 July 2014; revised 15 August 2014; accepted 25 August 2014

Copyright (C) 2014 by author and Scientific Research Publishing Inc.

This work is licensed under the Creative Commons Attribution International License (CC BY).

http://creativecommons.org/licenses/by/4.0/

(c) (i) Open Access

\begin{abstract}
The purpose of this paper is to introduce and study some sequence spaces which are defined by combining the concepts of sequences of Musielak-Orlicz functions, invariant means and lacunary convergence on 2-norm space. We establish some inclusion relations between these spaces under some conditions.
\end{abstract}

Keywords

Invariant Means, Musielak-Orlicz Functions, 2-Norm Space, Lacunary Sequence

\section{Introduction}

Let $\omega$ be the set of all sequences of real numbers $\ell_{\infty}, c$ and $c_{0}$ be respectively the Banach spaces of bounded, convergent and null sequences $x=\left(x_{k}\right)$ with $\left(x_{k}\right) \in \mathbb{R}$ or $\mathbb{C}$ the usual norm $\|x\|=\sup _{k}\left|x_{k}\right|$, where $k \in \mathbb{N}=1,2,3, \cdots$, the positive integers.

The idea of difference sequence spaces was first introduced by Kizmaz [1] and then the concept was generalized by Et and Çolak [2]. Later on Et and Esi [3] extended the difference sequence spaces to the sequence spaces:

$$
X\left(\Delta_{v}^{m}\right)=\left\{X=\left(x_{k}\right):\left(\Delta_{v}^{m} x\right) \in X\right\},
$$

for $X=\ell_{\infty}, c$ and $c_{0}$, where $v=\left(v_{k}\right)$ be any fixed sequence of non zero complex numbers and $\left(\Delta_{v}^{m} X_{k}\right)=\left(\Delta_{v}^{m-1} X_{k}-\Delta_{v}^{m-1} x_{k+1}\right)$.

The generalized difference operator has the following binomial representation, 


$$
\Delta_{v}^{m} X_{k}=\sum_{i=0}^{m}(-1)^{i}\left(\begin{array}{c}
m \\
i
\end{array}\right) v_{k+i} x_{k+i}, \text { for all } k \in \mathbb{N} .
$$

The sequence spaces $\Delta_{v}^{m}\left(\ell_{\infty}\right), \Delta_{v}^{m}(c)$ and $\Delta_{v}^{m}\left(c_{0}\right)$ are Banach spaces normed by

$$
\|x\|_{\Delta}=\sum_{i=1}^{m}\left|v_{i} x_{i}\right|+\left\|\Delta_{v}^{m} x\right\|_{\infty} .
$$

The concept of 2-normed space was initially introduces by Gahler [4] as an interesting linear generalization of normed linear space which was subsequently studied by many others [5] [6]). Recently a lot of activities have started to study summability, sequence spaces and related topics in these linear spaces [7] [8]).

Let $X$ be a real vector space of dimension $d$, where $2 \leq d<\infty$. A 2-norme on $X$ is a function $\|. .\|:. X \times X \rightarrow \mathbb{R}$ which satisfies:

1) $\|x, y\|=0$ if and only if $x$ and $y$ are linearly dependent,

2) $\|x, y\|=\|y, x\|$,

3) $\|\alpha x, y\|=|\alpha|\|x, y\|, \alpha \in \mathbb{R}$,

4) $\|x, y+z\| \leq\|x, y\|+\|x, z\|$.

The pair $(X,\|.,\|$.$) is called a 2-normed space. As an example of a 2-normed space we may take X=\mathbb{R}^{2}$ being equiped with the 2-norm $\|x, y\|=$ the area os paralelogram spaned by the vectors $x$ and $y$, which may be given explicitly by the formula

$$
\left\|x_{1}, x_{2}\right\|_{E}=a b s\left(\left|\begin{array}{ll}
x_{11} & x_{22} \\
x_{21} & x_{22}
\end{array}\right|\right) .
$$

Then clearly $(X,\|.,\|$.$) is 2-normed space. Recall that (X,\|.,\|$.$) is a 2-Banach space if every cauchy$ sequence in $X$ is convergent to some $x \in X$.

Let $\sigma$ be a mapping of the positive integers into itself. A continuous linear functional $\phi$ on $\ell_{\infty}$ is said to be an invariant mean or $\sigma$-mean if and only if

1) $\phi(x) \geq 0$, when the sequence $x=\left(x_{n}\right)$ has, $x_{n} \geq 0$ for all $n$,

2) $\phi(e)=1, e=(1,1,1, \cdots)$,

3) $\phi\left(x_{\sigma(n)}\right)=\phi(x)$ for all $x \in \ell_{\infty}$.

If $x=\left(x_{k}\right)$, where $T x=\left(T x_{k}\right)=\left(x_{\sigma(k)}\right)$. It can be shown that

$$
V_{\sigma}=\left\{x \in \ell_{\infty}: \lim _{k} t_{k n}(x)=l \text {, uniformly in } n\right\}
$$

$l=\sigma-\lim x$, where

$$
t_{k n}(x)=\frac{x_{n}+x_{\sigma^{1}(n)}+x_{\sigma^{2(n)}}+\cdots+x_{\sigma^{k(n)}}}{k+1} \text { [9]. }
$$

In the case $\sigma$ is the translation mapping $n \rightarrow n+1, \sigma$-mean is often called a Banach limit and $V_{\sigma}$ the set of bounded sequences of all whose invariant means are equal is the set of almost convergent sequence [10].

By Lacunary sequence $\theta=\left(k_{r}\right), r=0,1,2, \cdots$ where $k_{0}=0$ we mean an increasing sequence of non negative integers $h_{r}=\left(k_{r}-k_{r-1}\right) \rightarrow \infty(r \rightarrow \infty)$. The intervals determined by $\theta$ are denoted by $I_{r}=\left[k_{r-1}-k_{r}\right]$ and the ratio $\frac{k_{r}}{k_{r-1}}$ will be denoted by $q_{r}$. The space of lacunary strongly convergent sequence $N_{\theta}$ was defined by Freedman et al. [11] as follows:

$$
N_{\theta}=\left\{x=\left(x_{i}\right): \lim _{r \rightarrow \infty} \frac{1}{h_{r}} \sum_{k=I_{r}}\left|x_{k}-l\right|=0 \text { for some } \ell\right\} \text {. }
$$

An Orlicz function is a function $M:[0, \infty) \rightarrow[0, \infty)$ which is continuous, non-decreasing and convex with $M(0)=0, M(x)>0$ for $x>0$ and $M(x) \rightarrow \infty$ as $x \rightarrow \infty$. 
It is well known that if $M$ is convex function and $M(0)=0$ then $M(\lambda x) \leq \lambda M(x)$, for all $\lambda$ with $0 \leq \lambda \leq 1$.

Lindenstrauss and Tzafriri [12] used the idea of Orlicz function and defined the sequence space which was called an Orlicz sequence space $\ell_{M}$ such as

$$
\ell_{M}=\left\{x=\left(x_{k}\right): \sum_{k=1}^{\infty} M\left(\frac{\left|x_{k}\right|}{\rho}\right)<\infty, \text { for some } \rho>0\right\}
$$

which was a Banach space with the norm

$$
\|x\|=\inf \left\{\rho>0: \sum_{k=1}^{\infty} M\left(\frac{\left|x_{k}\right|}{\rho}\right) \leq 1\right\}
$$

which was called an Orlicz sequence space. The $\ell_{M}$ was closely related to the space $\ell_{p}$ which was an Orlicz sequence space with $M(t)=|t|^{p}$, for $1 \leq p<\infty$. Later the Orlicz sequence spaces were investigated by Prashar and Choudhry [13], Maddox [14], Tripathy et al. [15]-[17] and many others.

A sequence of function $M=\left(M_{k}\right)$ of Orlicz function is called a Musielak-Orlicz function [18] [19]. Also a Musielak-Orlicz function $\Phi=\left(\Phi_{k}\right)$ is called complementary function of a Musielak-Orlicz function $M$ if

$$
\Phi_{k}(t)=\sup \left\{|t| s-M_{k}(s): s \geq 0\right\}, \text { for } k=1,2,3, \cdots .
$$

For a given Musielak-Orlicz function $M$, the Musielak-Orlicz sequence space $l_{M}$ and its subspaces $\hbar_{M}$ are defined as follow:

$$
\begin{gathered}
l_{M}=\left\{x=x_{k} \in \omega: I_{M}(c x)<\infty, \text { for some } c>0\right\} \\
\hbar_{M}=\left\{x=x_{k} \in \omega: I_{M}(c x)<\infty, \text { for all } c>0\right\}
\end{gathered}
$$

where $I_{M}$ is a convex modular defined by

$$
I_{M}(x)=\sum_{k=1}^{\infty} M_{k}\left(x_{k}\right), x=\left(x_{k}\right) \in l_{M} .
$$

We consider $l_{M}$ equipped with the Luxemburg norm

$$
\|x\|=\inf \left\{k>0: I_{M}\left(\frac{x}{k}\right) \leq 1\right\}
$$

or equipped with the Orlicz norm

$$
\|x\|^{0}=\inf \left\{\frac{1}{k}\left(1+I_{M}(k x)\right): k>0\right\} .
$$

The main purpose of this paper is to introduce the following sequence spaces and examine some properties of the resulting sequence spaces. Let $M=\left(M_{k}\right)$ be a Musielak-Orlicz function, $(X,\|, .\|$,$) is called a 2-normed$ space. Let $p=\left(p_{k}\right)$ be any sequences of positive real numbers, for all $k \in \mathbb{N}$ and $u=\left(u_{k}\right)$ such that $u_{k} \neq 0(k=1,2,3, \cdots)$. Let $s$ be any real number such that $s \geq 0$. By $\omega(2-X)$ we denote the space of all sequences defined over $(X,\|.\|$,$) . Then we define the following sequence spaces:$

$$
\begin{aligned}
& {\left[\omega^{\theta}, M, p, u, s,\|, . .\|_{\sigma}^{\infty}\left(\Delta_{v}^{m}\right)\right.} \\
& =\left\{x=\left(x_{k}\right) \in \omega(2-X): \sup _{r, n} \frac{1}{h_{r}} \sum_{k \in I_{r}} k^{-s} u_{k}\left[M_{k}\left(\left\|\frac{t_{k n}\left(\Delta_{v}^{m} x_{k}\right)}{\rho}, z\right\|\right)\right]^{p_{k}}<\infty \rho>0, s \geq 0\right\}
\end{aligned}
$$




$$
\begin{aligned}
& {\left[\omega^{\theta}, M, p, u, s,\|, .,\|\right]_{\sigma}\left(\Delta_{v}^{m}\right)} \\
& =\left\{x=\left(x_{k}\right) \in \omega(2-X):: \lim _{r} \frac{1}{h_{r}} \sum_{k \in I_{r}} k^{-s} u_{k}\left[M_{k}\left(\left\|\frac{t_{k n}\left(\Delta_{v}^{m} x_{k}\right)}{\rho}, z\right\|\right)\right]^{p_{k}}=0 \text { for some } l, \rho>0, s \geq 0\right\} \\
& {\left[\omega^{\theta}, M, p, u, s,\|, .\|\right]_{\sigma}^{0}\left(\Delta_{v}^{m}\right)} \\
& =\left\{x=\left(x_{k}\right) \in \omega(2-X):: \lim _{r} \frac{1}{h_{r}} \sum_{k \in I_{r}} k^{-s} u_{k}\left[M_{k}\left(\left\|\frac{t_{k n}\left(\Delta_{v}^{m} x_{k}\right)}{\rho}, z\right\|\right)\right]^{p_{k}}=0 \rho>0, s \geq 0\right\}
\end{aligned}
$$

Definition 1. A sequence space $E$ is said to be solid or normal if $\left(\alpha_{k} x_{k}\right) \in E$ whenever $\left(x_{k}\right) \in E$ and for all sequences of scalar $\left(\alpha_{k}\right)$ with $\left|\alpha_{k}\right| \leq 1$ [20].

Definition 2. A sequence space $E$ is said to be monotone if it contains the canonical pre-images of all its steps spaces, [20].

Definition 3. If $X$ is a Banach space normed by $\|\cdot\|$, then $\Delta^{m}(X)$ is also Banach space normed by

$$
\|x\|_{\Delta}=\sum_{k=1}^{m}\left|x_{k}\right|+f\left(\Delta^{m} x\right) \text {. }
$$

Remark 1. The following inequality will be used throughout the paper. Let $p=\left(p_{k}\right)$ be a positive sequence of real numbers with $0<p_{k} \leq \sup p_{k}=G, D=\max \left(1,2^{G-1}\right)$. Then for all $a_{k}, b_{k} \in \mathbb{C}$ for all $k \in \mathbb{N}$. We have

$$
\left|a_{k}+b_{k}\right|^{p_{k}} \leq D\left(\left|a_{k}\right|^{p_{k}}+\left|b_{k}\right|^{p_{k}}\right) \text {. }
$$

\section{Main Results}

Theorem 1. Let $M=\left(M_{k}\right)$ be a Musielak-Orlicz function, $p=\left(p_{k}\right)$ be a bounded sequence of positive real number and $\theta=\left(k_{r}\right)$ be a lacunary sequence. Then $\left[\omega^{\theta}, M, p, u, s,\|.,\|\right]_{\sigma}^{\infty}\left(\Delta_{v}^{m}\right),\left[\omega^{\theta}, M, p, u, s,\|, .,\|\right]_{\sigma}\left(\Delta_{v}^{m}\right)$ and $\left[\omega^{\theta}, M, p, u, s,\|, .,\|\right]_{\sigma}^{0}\left(\Delta_{v}^{m}\right)$ are linear spaces over the field of complex numbers.

Proof 1. Let $x=\left(x_{k}\right), y=\left(y_{k}\right) \in\left[\omega^{\theta}, M, p, u, s,\|, .,\|\right]_{\sigma}^{0}\left(\Delta_{v}^{m}\right)$ and $\alpha, \beta \in \mathbf{C}$. In order to prove the result we need to find some $\rho_{3}$ such that,

$$
\lim _{r \rightarrow \infty} \frac{1}{h_{r}} \sum_{k \in I_{r}} u_{k} k^{-s}\left[M_{k}\left(\left\|\frac{\left|t_{n k}\left(\Delta_{v}^{m}\left(\alpha x_{k}+\beta y_{k}\right)\right)\right|}{\rho_{3}}, z\right\|\right)\right]^{p_{k}}=0 \text {, uniformly in } n .
$$

Since $\left(x_{k}\right),\left(y_{k}\right) \in\left[\omega^{\theta}, M, p, u, s,\|, \cdot,\|\right]_{\sigma}^{0}\left(\Delta_{v}^{m}\right)$, there exist positive $\rho_{1}, \rho_{2}$ such that

$$
\lim _{r \rightarrow \infty} \frac{1}{h_{r}} \sum_{k \in I_{r}} u_{k} k^{-s}\left[M_{k}\left(\left\|\frac{t_{k n}\left(\Delta_{v}^{m} x_{k}\right)}{\rho}, z\right\|\right)\right]^{p_{k}}=0 \text { uniformly in } n
$$

and

$$
\lim _{r \rightarrow \infty} \frac{1}{h_{r}} \sum_{k \in I_{r}} u_{k} k^{-s}\left[M_{k}\left(\left\|\frac{t_{k n}\left(\Delta_{v}^{m} x_{k}\right)}{\rho}, z\right\|\right)\right]^{p_{k}}=0 \text { uniformly in } n .
$$

Define $\rho_{3}=\max \left(2|\alpha| \rho_{1}, 2|\beta| \rho_{2}\right)$. Since $\left(M_{k}\right)$ is non decreasing and convex 


$$
\begin{aligned}
& \frac{1}{h_{r}} \sum_{k \in I_{r}} u_{k} k^{-s}\left[M_{k}\left(\left\|\frac{t_{n k}\left(\Delta_{v}^{m}\left(\alpha x_{k}+\beta y_{k}\right)\right)}{\rho_{3}}, z\right\|\right)\right]^{p_{k}} \\
& \leq \frac{1}{h_{r}} \sum_{k \in I_{r}} u_{k} k^{-s}\left[M_{k}\left(\left\|\frac{t_{n k}\left(\Delta_{v}^{m}\left(\alpha x_{k}\right)\right)}{\rho_{3}}, z\right\|+\left\|\frac{t_{n k}\left(\Delta_{v}^{m}\left(\beta y_{k}\right)\right)}{\rho_{3}}, z\right\|\right)\right]^{p_{k}} \\
& \left.\leq \frac{1}{h_{r}} \sum_{k \in I_{r}} u_{k} k^{-s}\left[M_{k}\left(\left\|\frac{t_{n k}\left(\Delta_{v}^{m} x_{k}\right)}{\rho_{1}}, z\right\|+\left\|\frac{t_{n k}\left(\Delta_{v}^{m} y_{k}\right)}{\rho_{2}}, z\right\|\right)\right]^{p_{k}} \|\right]^{p_{k}}+\frac{D}{h_{r}} \sum_{k \in I_{r}} u_{k} k^{-s}\left[M_{k}\left(\left\|\frac{t_{n k}\left(\Delta_{v}^{m} y_{k}\right)}{\rho}, z\right\|\right)\right]^{p_{k}} \\
& \leq \frac{D}{h_{r}} \sum_{k \in I_{r}} u_{k} k^{-s}\left[M_{k}\left(\left\|\frac{t_{n k}\left(\Delta_{v}^{m} x_{k}\right)}{\rho}, z\right\|\right)\right]^{\rho_{k}} \| \text { as } r \rightarrow \infty \text {, uniformly in n. }
\end{aligned}
$$

So that $\left(\alpha x_{k}\right)+\left(\beta y_{k}\right) \in\left[\omega^{\theta}, M, p, u, s,\|.,\|\right]_{\sigma}^{0}\left(\Delta_{v}^{m}\right)$. This completes the proof. Similarly, we can prove that $\left[\omega^{\theta}, M, p, u, s,\|.,\|\right]_{\sigma}\left(\Delta_{v}^{m}\right)$ and $\left[\omega^{\theta}, M, p, u, s,\|.,\|\right]_{\sigma}^{\infty}\left(\Delta_{v}^{m}\right)$ are linear spaces.

Theorem 2. Let $M=\left(M_{k}\right)$ be a Musielak-Orlicz function, $p=\left(p_{k}\right)$ be a bounded sequence of positive real number and $\theta=\left(k_{r}\right)$ be a lacunary sequence. Then $\left[\omega^{\theta}, M, p, u, s,\|, \cdot\|\right]_{\sigma}^{0}\left(\Delta_{v}^{m}\right)$ is a topological linear space totalparanormed by

$$
g_{\Delta}(x)=\sum_{k=1}^{m}\left|x_{k}\right|+\inf \left\{\rho^{p_{r} / H}:\left(\frac{1}{h_{r}} \sum_{k \in I_{r}} u_{k} k^{-s}\left[M_{k}\left(\left\|\frac{t_{n k}\left(\Delta_{v}^{m} x_{k}\right)}{\rho}, z\right\|\right)\right]^{p_{k}}\right]^{1 / H} \leq 1 \text { for some } \rho, r=1,2, \cdots\right\}
$$

Proof 2. Clearly $g_{\Delta}(x)=g_{\Delta}(-x)$. Since $M_{k}(0)=0$, for all $k \in \mathbb{N}$. we get $g_{\Delta}(\bar{\theta})=0$, for $x=\bar{\theta}$. Let $x=\left(x_{k}\right), y=\left(y_{k}\right) \in\left[\omega^{\theta}, M, p, u, s,\|, .,\|\right]_{\sigma}^{0}\left(\Delta_{v}^{m}\right)$ and let us choose $\rho_{1}>0$ and $\rho_{2}>0$ such that

$$
\sup _{r} h_{r}^{-1} \sum_{k \in I_{r}} u_{k} k^{-s}\left[M_{k}\left(\left\|\frac{t_{n k}\left(\Delta_{v}^{m}\left(x_{k}\right)\right)}{\rho_{1}}, z\right\|\right)\right]^{p_{k}} \leq 1 \quad r=1,2,3, \cdots
$$

and

$$
\sup _{r} h_{r}^{-1} \sum_{k \in I_{r}} u_{k} k^{-s}\left[M_{k}\left(\left\|\frac{t_{n k}\left(\Delta_{v}^{m}\left(y_{k}\right)\right)}{\rho_{2}}, z\right\|\right)\right]^{p_{k}} \leq 1 \quad r=1,2,3, \cdots
$$

Let $\rho=\rho_{1}+\rho_{2}$, then we have

$$
\begin{aligned}
& \sup _{r} h_{r}^{-1} \sum_{k \in I_{r}} u_{k} k^{-s}\left[M_{k}\left(\left\|\frac{t_{n k}\left(\Delta_{v}^{m}\left(x_{k}+y_{k}\right)\right)}{\rho}, z\right\|\right)\right]^{p_{k}} \\
& \leq \frac{\rho_{1}}{\rho_{1}+\rho_{2}} \sup _{r} h_{r}^{-1} \sum_{k \in I_{r}} u_{k} k^{-s}\left[M_{k}\left(\left\|\frac{t_{n k}\left(\Delta_{v}^{m}\left(x_{k}\right)\right)}{\rho_{1}}, z\right\|\right)\right]^{p_{k}} \\
& +\frac{\rho_{1}}{\rho_{1}+\rho_{2}} \sup _{r} h_{r}^{-1} \sum_{k \in I_{r}} u_{k} k^{-s}\left[M_{k}\left(\left\|\frac{t_{n k}\left(\Delta_{v}^{m}\left(y_{k}\right)\right)}{\rho_{2}}, z\right\|\right)\right]^{p_{k}} \leq 1 .
\end{aligned}
$$


Since $\rho>0$, we have

$$
\begin{aligned}
& g_{\Delta}(x+y) \\
& =\sum_{k=1}^{m}\left|x_{k}+y_{k}\right|+\inf \left\{\rho^{p_{r} / H}:\left(\frac{1}{h_{r}} \sum_{k \in I_{r}} u_{k} k^{-s}\left[M_{k}\left(\left\|\frac{t_{n k} \Delta_{v}^{m}\left(x_{k}+y_{k}\right)}{\rho}, z\right\|\right)\right]^{p_{k}}\right)^{1 / H} \leq 1 \text { for some } \rho>0, r=1,2, \cdots\right\} \\
& \leq \sum_{k=1}^{m}\left|x_{k}\right|+\inf \left\{\rho_{1}^{p_{r} / H}:\left(\frac{1}{h_{r}} \sum_{k \in I_{r}} u_{k} k^{-s}\left[M_{k}\left(\left\|\frac{t_{n k} \Delta_{v}^{m}\left(x_{k}\right)}{\rho_{1}}, z\right\|\right)\right]^{p_{k}}\right)^{1 / H} \leq 1 \text { for some } \rho_{1}>0, r=1,2, \cdots\right\} \\
& +\sum_{k=1}^{m}\left|y_{k}\right|+\inf \left\{\rho_{2}^{p_{r} / H}:\left(\frac{1}{h_{r}} \sum_{k \in I_{r}} u_{k} k^{-s}\left[M_{k}\left(\left\|\frac{t_{n k} \Delta_{v}^{m}\left(y_{k}\right)}{\rho_{2}}, z\right\|\right)^{p_{k}}\right)^{1 / H} \leq 1 \text { for some } \rho_{2}>0, r=1,2, \cdots\right\} .\right. \\
& g_{\Delta}(x+y) \leq g_{\Delta}(x)+g_{\Delta}(y) .
\end{aligned}
$$

Finally, we prove that the scalar multiplication is continuous. Let $\lambda$ be a given non zero scalar in $\mathbb{C}$. Then the continuity of the product follows from the following expression.

$$
\begin{aligned}
g_{\Delta}(\lambda x) & =\sum_{k=1}^{m}\left|\lambda x_{k}\right|+\inf \left\{\rho^{p_{r} / H}:\left(\frac{1}{h_{r}} \sum_{k \in I_{r}} u_{k} k^{-s}\left[M_{k}\left(\left\|\frac{t_{n k} \Delta_{v}^{m}\left(\lambda x_{k}\right)}{\rho}, z\right\|\right)\right]^{p_{k}}\right)^{1 / H} \leq 1 \text { for some } \rho>0, r=1,2, \cdots\right\} \\
& =\lambda \sum_{k=1}^{m}\left|x_{k}\right|+\inf \left\{(|\lambda| \zeta)^{p_{r} / H}:\left(\frac{1}{h_{r}} \sum_{k \in I_{r}} u_{k} k^{-s}\left[M_{k}\left(\left\|\frac{t_{n k} \Delta_{v}^{m}\left(x_{k}\right)}{\zeta}, z\right\|\right)\right]^{p_{k}}\right)^{1 / H} \leq 1 \text { for some } \zeta>0, r=1,2, \cdots\right\}
\end{aligned}
$$

where $\zeta=\frac{\rho}{|\lambda|}>0$. Since $|\lambda|^{p_{r}} \leq \max (1,|\lambda|)^{\text {sup } p_{r}}$,

$$
\begin{aligned}
& g_{\Delta}(\lambda x)=\max (1,|\lambda|)^{\text {sup } p_{r}} \\
& +\inf \left\{\rho^{p_{r} / H}:\left(\frac{1}{h_{r}} \sum_{k \in I_{r}} u_{k} k^{-s}\left[M_{k}\left(\left\|\frac{t_{n k}\left(\Delta_{v}^{m} x_{k}\right)}{\rho}, z\right\|\right)\right]^{p_{k}}\right)^{1 / H} \leq 1, \text { for some } \rho>0, r=1,2, \cdots\right\} .
\end{aligned}
$$

This completes the proof of this theorem.

Theorem 3. Let $M=\left(M_{k}\right)$ be a Musielak-Orlicz function, $p=\left(p_{k}\right)$ be a bounded sequence of positive real number and $\theta=\left(k_{r}\right)$ be a lacunary sequence. Then

$\left[\omega^{\theta}, M, p, u, s,\|, .,\|\right]_{\sigma}^{\infty}\left(\Delta_{v}^{m}\right) \subset\left[\omega^{\theta}, M, p, u, s,\|, .,\|\right]_{\sigma}\left(\Delta_{v}^{m}\right) \subset\left[\omega^{\theta}, M, p, u, s,\|, .,\|\right]_{\sigma}^{0}\left(\Delta_{v}^{m}\right)$.

Proof 3. The inclusion $\left[\omega^{\theta}, M, p, u, s,\|, \cdot\|\right]_{\sigma}^{0}\left(\Delta_{v}^{m}\right) \subset\left[\omega^{\theta}, M, p, u, s,\|., .\|\right]_{\sigma}\left(\Delta_{v}^{m}\right)$ is obvious. Let $x_{k} \in\left[\omega^{\theta}, M, p, u, s,\|,\|\right]_{\sigma}\left(\Delta_{v}^{m}\right)$. Then there exists some positive number $\rho_{1}$ such that

$$
\lim _{r \rightarrow \infty} \frac{1}{h_{r}} \sum_{k \in I_{r}} u_{k} k^{-s}\left[M_{k}\left(\left\|\frac{t_{n k}\left(\Delta_{v}^{m} x_{k}-l e\right)}{\rho_{1}}, z\right\|\right)\right]^{p_{k}} \rightarrow 0
$$

as $r \rightarrow \infty$, uniformly in $n$. Define $\rho=2 \rho_{1}$. Since $M_{k}$ is non decreasing and convex for all $k \in \mathbb{N}$, we have 


$$
\begin{aligned}
& \frac{1}{h_{r}} \sum_{k \in I_{r}} u_{k} k^{-s}\left[M_{k}\left(\left\|\frac{t_{n k}\left(\Delta_{v}^{m} x_{k}\right)}{\rho}, z\right\|\right)\right]^{p_{k}} \\
& \leq \frac{D}{h_{r}} \sum_{k \in I_{r}} u_{k} k^{-s}\left[M_{k}\left(\left\|\frac{t_{n k}\left(\Delta_{v}^{m} x_{k}-l e\right)}{\rho_{1}}, z\right\|\right)\right]^{p_{k}} \\
& +\frac{D}{h_{r}} \sum_{k \in I_{r}}\left[M_{k}\left(\left\|\frac{l e}{\rho_{1}}, z\right\|\right)\right]^{p_{k}} \\
& \leq \frac{D}{h_{r}} \sum_{k \in I_{r}}\left[M_{k}\left(\left\|\frac{t_{n k}\left(\Delta_{v}^{m} x_{k}-l e\right)}{\rho_{1}}, z\right\|\right)\right]^{p_{k}} \\
& +D \max \left\{1,\left[M\left(\left\|\frac{l e}{\rho_{1}}, \mathrm{z}\right\|\right)\right]^{G}\right\}
\end{aligned}
$$

where $G=\sup _{k}\left(p_{k}\right), D=\max \left(1,2^{G}-1\right)$ by (1).

Thus $x_{k} \in\left[\omega^{\theta}, M, p, u, s,\|., .\|\right]_{\sigma}\left(\Delta_{v}^{m}\right)$.

Theorem 4. Let $M=\left(M_{k}\right)$ be a Musielak-Orlicz functions. If $\sup _{k}\left[M_{k}(z)\right]^{p_{k}}<\infty$ for all $z>0$, then $\left[\omega^{\theta}, M, p, u, s,\|., .\|\right]_{\sigma}\left(\Delta_{v}^{m}\right) \subset\left[\omega^{\theta}, M, p, u, s, \| ., .\right]_{\sigma}^{\infty}\left(\Delta_{v}^{m}\right)$.

Proof 4. Let $x_{k} \in\left[\omega^{\theta}, M, p, u, s, \| ., .\right]_{\sigma}\left(\Delta_{v}^{m}\right)$ by using (1), we have

$$
\begin{aligned}
& \frac{1}{h_{r}} \sum_{k \in I_{r}} u_{k} k^{-s}\left[M_{k}\left(\left\|\frac{t_{n k}\left(\Delta_{v}^{m} x_{k}\right)}{\rho}, z\right\|\right)\right]^{p_{k}} \\
& \leq \frac{D}{h_{r}} \sum_{k \in I_{r}}\left[M_{k}\left(\left\|\frac{t_{n k}\left(\Delta_{v}^{m} x_{k}-l e\right)}{\rho}, z\right\|\right)\right]^{p_{k}} \\
& +\frac{D}{h_{r}} \sum_{k \in I_{r}}\left[M_{k}\left(\left\|\frac{|e|}{\rho}, z\right\|\right)\right]^{p_{k}} .
\end{aligned}
$$

Since $\sup _{k}[M(z)]^{p_{k}}<\infty$, we can take the $\sup _{k}[M(z)]^{p_{k}}=K$. Hence we can get $x_{k} \in\left[\omega^{\theta}, M, p, u, s,\|., .\|\right]_{\sigma}\left(\Delta_{v}^{m}\right)$.

This complete the proof.

Theorem 5. Let $m \geq 1$ be fixed integer. Then the following statements are equivalent:

1) $\left[\omega^{\theta}, M, p, u, s,\|., .\|\right]_{\sigma}^{\infty}\left(\Delta_{v}^{m-1}\right) \subset\left[\omega^{\theta}, M, p, u, s,\|., .\|\right]_{\sigma}^{\infty}\left(\Delta_{v}^{m}\right)$,

2) $\left[\omega^{\theta}, M, p, u, s,\|., .\|\right]_{\sigma}\left(\Delta_{v}^{m-1}\right) \subset\left[\omega^{\theta}, M, p, u, s,\|., .\|\right]_{\sigma}\left(\Delta_{v}^{m}\right)$,

3) $\left[\omega^{\theta}, M, p, u, s,\|., .\|\right]_{\sigma}^{0}\left(\Delta_{v}^{m-1}\right) \subset\left[\omega^{\theta}, M, p, u, s,\|., .\|\right]_{\sigma}^{0}\left(\Delta_{v}^{m}\right)$.

Proof 5. Let $x_{k} \in\left[\omega^{\theta}, M, p, u, s,\|., .\|\right]_{\sigma}^{0}\left(\Delta_{v}^{m-1}\right)$. Then there exist $\rho>0$ such that

$$
\lim _{r \rightarrow \infty} \frac{1}{h_{r}} \sum_{k \in I_{r}} u_{k} k^{-s}\left[M_{k}\left(\left\|\frac{t_{n k}\left(\Delta_{v}^{m} x_{k}\right)}{\rho}, z\right\|\right)\right]^{p_{k}} \rightarrow 0 .
$$

Since $M_{k}$ is non decreasing and convex, we have 
M. Aiyub

$$
\begin{aligned}
& \frac{1}{h_{r}} \sum_{k \in I_{r}} u_{k} k^{-s}\left[M_{k}\left(\left\|\frac{t_{n k}\left(\Delta_{v}^{m} x_{k}\right)}{2 \rho}, z\right\|\right)\right]^{p_{k}} \\
& =\frac{1}{h_{r}} \sum_{k \in I_{r}} u_{k} k^{-s}\left[M_{k}\left(\left\|\frac{\| t_{n k}\left(\Delta_{v}^{m-1} x_{k}-\Delta^{m-1} x_{k+1}\right) \mid}{2 \rho}, z\right\|\right)\right]^{p_{k}} \\
& \leq \frac{1}{h_{r}} \sum_{k \in I_{r}} u_{k} k^{-s}\left[M_{k}\left(\left\|\frac{\| t_{n k}\left(\Delta_{v}^{m-1} x_{k}\right)}{2 \rho}, z\right\|\right)\right]^{p_{k}}+\frac{1}{h_{r}} \sum_{k \in I_{r}} u_{k} k^{-s}\left[M_{k}\left(\left\|\frac{\| t_{n k}\left(\Delta_{v}^{m-1} x_{k+1}\right)}{2 \rho}, z\right\|\right)\right]^{p_{k}} \\
& \leq \frac{D}{h_{r}} \sum_{k \in I_{r}} u_{k} k^{-s}\left[M_{k}\left(\left\|\frac{t_{n k}\left(\Delta_{v}^{m-1} x_{k}\right)}{\rho}, z\right\|\right)\right]^{p_{k}}+\frac{D}{h_{r}} \sum_{k \in I_{r}} u_{k} k^{-s}\left[M_{k}\left(\left\|\frac{t_{n k}\left(\Delta_{v}^{m-1} x_{k+1}\right)}{\rho}, z\right\|\right)\right]^{p_{k}} .
\end{aligned}
$$

Taking $\lim _{r \rightarrow \infty}$, we have

$$
\frac{1}{h_{r}} \sum_{k \in I_{r}} u_{k} k^{-s}\left[M_{k}\left(\left\|\frac{t_{n k}\left(\Delta_{v}^{m} x_{k}\right)}{\rho}, z\right\|\right)\right]^{p_{k}}=0,
$$

ie. $\quad x_{k} \in\left[\omega^{\theta}, M, p, u, s, \| ., .\right]_{\sigma}^{0}\left(\Delta_{v}^{m-1}\right)$. The rest of these cases can be proved in similar way.

Theorem 6. Let $M=\left(M_{k}\right)$ and $T=\left(T_{k}\right)$ be two Musielak-Orlicz functions. Then we have

1) $\left[\omega^{\theta}, M, p, u, s,\|, .,\|\right]_{\sigma}^{\infty}\left(\Delta_{v}^{m}\right) \cap\left[\omega^{\theta}, T, p, u, s,\|, .,\|\right]_{\sigma}^{\infty}\left(\Delta_{v}^{m}\right) \subset\left[\omega^{\theta}, M+T, p, u, s,\|, .,\|\right]_{\sigma}^{\infty}\left(\Delta_{v}^{m}\right)$.

2) $\left[\omega^{\theta}, M, p, u, s,\|, .\|\right]_{\sigma}\left(\Delta_{v}^{m}\right) \cap\left[\omega^{\theta}, T, p, u, s,\|, .\|\right]_{\sigma}\left(\Delta_{v}^{m}\right) \subset\left[\omega^{\theta}, M+T, p, u, s,\|, .,\|\right]_{\sigma}\left(\Delta_{v}^{m}\right)$.

3) $\left[\omega^{\theta}, M, p, u, s,\|, .,\|\right]_{\sigma}^{0}\left(\Delta_{v}^{m}\right) \cap\left[\omega^{\theta}, T, p, u, s,\|, .,\|\right]_{\sigma}^{0}\left(\Delta_{v}^{m}\right) \subset\left[\omega^{\theta}, M+T, p, u, s,\|, .,\|\right]_{\sigma}^{0}\left(\Delta_{v}^{m}\right)$.

Proof 6. Let $x_{k} \in\left[\omega^{\theta}, M, p, u, s,\|.,\|\right]_{\sigma}^{\infty}\left(\Delta_{v}^{m}\right) \cap\left[\omega^{\theta}, T, p, u, s,\|.,\|\right]_{\sigma}^{\infty}\left(\Delta_{v}^{m}\right)$. Then

$$
\sup _{r, n} \frac{1}{h_{r}} \sum_{k \in I_{r}} u_{k} k^{-s}\left[M_{k}\left(\left\|\frac{t_{n k}\left(\Delta_{v}^{m} x_{k}\right)}{\rho}, z\right\|\right)\right]^{p_{k}}<\infty
$$

and

$$
\sup _{r, n} \frac{1}{h_{r}} \sum_{k \in I_{r}} u_{k} k^{-s}\left[T_{k}\left(\left\|\frac{t_{n k}\left(\Delta_{v}^{m} x_{k}\right)}{\rho}, z\right\|\right)\right]^{p_{k}}<\infty
$$

uniformly in $n$. We have

$$
\left[\left(M_{k}+T_{k}\right)\left(\left\|\frac{t_{n k}\left(\Delta_{v}^{m} x_{k}\right)}{\rho}, z\right\|\right)\right]^{p_{k}} \leq D\left[M_{k}\left(\left\|\frac{t_{n k}\left(\Delta_{v}^{m} x_{k}\right)}{\rho}, z\right\|\right)\right]^{p_{k}}+D\left[T_{k}\left(\left\|\frac{t_{n k}\left(\Delta_{v}^{m} x_{k}\right)}{\rho}, z\right\|\right)\right]^{p_{k}}
$$

by (1). Applying $\sum_{k \in I_{r}}$ and multiplying by $u_{k}, \frac{1}{h_{r}}$ and $k^{-s}$ both side of this inequality, we get

$$
\begin{aligned}
& \frac{1}{h_{r}} \sum_{k \in I_{r}} u_{k} k^{-s}\left[\left(M_{k}+T_{k}\right)\left(\left\|\frac{t_{n k}\left(\Delta_{v}^{m} x_{k}\right)}{\rho}, z\right\|\right)\right]^{p_{k}} \\
& \leq \frac{D}{h_{r}} \sum_{k \in I_{r}} u_{k} k^{-s}\left[M_{k}\left(\left\|\frac{t_{n k}\left(\Delta_{v}^{m} x_{k}\right)}{\rho}, z\right\|\right)^{p_{k}}+\frac{D}{h_{r}} \sum_{k \in I_{r}} u_{k} k^{-s}\left[T_{k}\left(\left\|\frac{t_{n k}\left(\Delta_{v}^{m} x_{k}\right)}{\rho}, z\right\|\right)\right]^{p_{k}}\right.
\end{aligned}
$$

2609 
uniformly in $n$. This completes the proof 2) and 3) can be proved similar to 1).

Theorem 7. 1) The sequence spaces $\left[\omega^{\theta}, M, p, u, s,\|.,\|\right]_{\sigma}^{\infty}$ and $\left[\omega^{\theta}, M, p, u, s,\|, .,\|\right]_{\sigma}^{0}$ are solid and hence they are monotone.

2) The space $\left[\omega^{\theta}, M, p, u, s,\|., .\|\right]_{\sigma}$ is not monotone and neither solid nor perfect.

Proof 7. We give the proof for $\left[\omega^{\theta}, M, p, u, s,\|, .,\|\right]_{\sigma}^{0}$. Let $x_{k} \in\left[\omega^{\theta}, M, p, u, s,\|, .,\|\right]_{\sigma}^{0}$ and $\left(\alpha_{k}\right)$ be a sequence of scalars such that $\left|\alpha_{k}\right| \leq 1$ for all $k \in \mathbb{N}$. Then we have

$$
\frac{1}{h_{r}} \sum_{k \in I_{r}} u_{k} k^{-s}\left[M_{k}\left(\left\|\frac{t_{n k}\left(\alpha_{k} x_{k}\right)}{\rho}, z\right\|\right)\right]^{p_{k}} \leq \frac{1}{h_{r}} \sum_{k \in I_{r}} u_{k} k^{-s}\left[M_{k}\left(\left\|\frac{t_{n k}\left(x_{k}\right)}{\rho}, z\right\|\right)\right]^{p_{k}} \rightarrow 0
$$

$(r \rightarrow \infty)$, uniformly in $n$. Hence $\left(\alpha_{k} x_{k}\right) \in\left[\omega^{\theta}, M, p, u, s,\|, .\|\right]_{\sigma}^{0}$ for all sequence of scalars $\left(\alpha_{k}\right)$ with $\left|\alpha_{k}\right| \leq 1$ for all $k \in \mathbb{N}$, whenever $x_{k} \in\left[\omega^{\theta}, M, p, u, s,\|,\|\right]_{\sigma}^{0}$. The spaces are monotone follows from the remark (1).

\section{References}

[1] Kizmaz, H. (1981) On Certain Sequence Spaces. Canadian Mathematical Bulletin, 24, 169-176. http://dx.doi.org/10.4153/CMB-1981-027-5

[2] Et, M. and Çolak, R. (1995) On Some Generalized Difference Sequence Spaces. Soochow Journal of Mathematics, 21, 377-386.

[3] Et, M. and Esi, A. (2000) On Köthe-Toeplitz Duals of Generalized Difference Sequence. Bulletin of the Malaysian Mathematical Society, 23, 25-32.

[4] Gahler, S. (1963) 2-Metrische Raume Undihre Topologishe Struktur. Mathematische Nachrichten, 26, 115-148. http://dx.doi.org/10.1002/mana.19630260109

[5] Gunawan, H. and Mashadi, H. (2001) On Finite Dimensional 2-Normed Spaces. Soochow Journal of Mathematics, 27, 321-329.

[6] Freese, R.W. and Cho, Y.J. (2001) Geometry of Linear 2-Normed Spaces. Nova Science Publishers, Huntington.

[7] Gurdal, M. and Pehlivan, S. (2009) Statitical Convergence in 2-Normed Spaces. Southeast Asian Bulletin of Mathematics, 33, 257-264.

[8] Sahiner, A., Gurda, M., Saltan, S. and Gunawan, H. (2007) Ideal Convergence in 2-Normed Spaces. Taiwanese Journal of Mathematics, 11, 1477-1484.

[9] Schaefer, P. (1972) Infinite Matrices and Invariant Means. Proceedings of the American Mathematical Society, 36, 104-110. http://dx.doi.org/10.1090/S0002-9939-1972-0306763-0

[10] Lorenz, G.G. (1948) A Contribution to the Theory of Divergent Sequences. Acta Mathematica, 80, 167-190. http://dx.doi.org/10.1007/BF02393648

[11] Freedman, A.R., Sember, J.J. and Raphael, R. (1978) Some p-Cesáo-Type Summability Spaces. Proceedings of the London Mathematical Society, S3-37, 508-520. http://dx.doi.org/10.1112/plms/s3-37.3.508

[12] Lindendstrauss, J. and Tzafriri, L. (1972) On Orlicz Sequence Spaces. Israel Journal of Mathematics, 10, 379-390. http://dx.doi.org/10.1007/BF02771656

[13] Prashar, S.D. and Choudhry, B. (1995) Sequence Spaces Defined by Orlicz Functions. Indian Journal of Pure and Applied Mathematics, 25, 419-428.

[14] Maddox, I.J. (1976) Spaces of Strongly Summable Sequences. Quarterly Journal of Mathematics, 18, 345-355. http://dx.doi.org/10.1093/qmath/18.1.345

[15] Tripathy, B.C., Et, M., Altin, Y. and Choudhry, B. (2003) On Some Class of Sequences Defined by Sequence of Orlicz Functions. Journal of Analysis and Applications, 1, 175-192.

[16] Tripathy, B.C. and Mohanta, S. (2004) On a Class of Generalized Lacunary Difference Sequence Spaces Defined by Orlicz Functions. Acta Mathematicae Applicatae Sinica, 20, 231-238. http://dx.doi.org/10.1007/s10255-004-0163-1

[17] Tripathy, B.C., Mohanta, S. and Et, M. (2005) On Generalized Lacunary Difference Vector Valued Paranormed Sequences Defined by Orlicz Functions. International Journal of Mathematical Sciences, 4, 341-355. 
[18] Maligranda, L. (1989) Orlicz Spaces and Interpolation, Seminars in Mathematics 5. Polish Academy of Science, Warsaw.

[19] Musielak, J. (1983) Orlicz Spaces and Modular Spaces. Lecture Notes in Mathematics, 1034.

[20] Kamthan, P.K. and Gupta, M. (1981) Sequence Spaces and Series. Marcel Dekker, Inc., New York. 
Scientific Research Publishing (SCIRP) is one of the largest Open Access journal publishers. It is currently publishing more than 200 open access, online, peer-reviewed journals covering a wide range of academic disciplines. SCIRP serves the worldwide academic communities and contributes to the progress and application of science with its publication.

Other selected journals from SCIRP are listed as below. Submit your manuscript to us via either submit@scirp.org or Online Submission Portal.
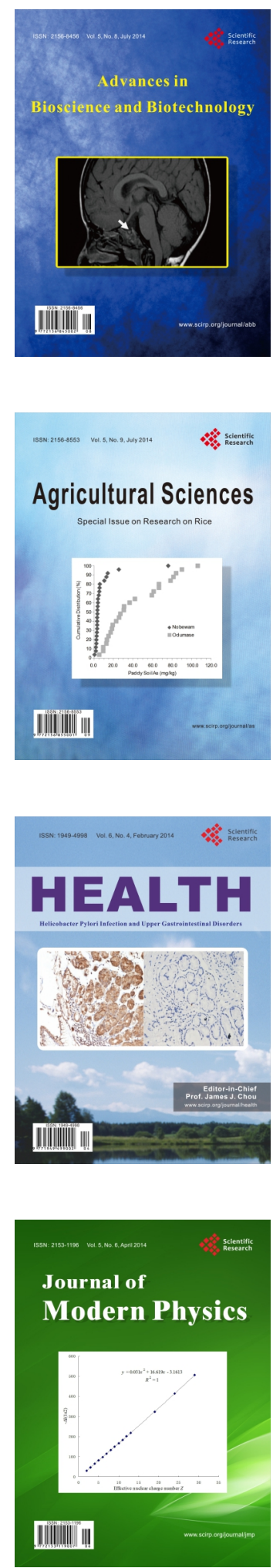
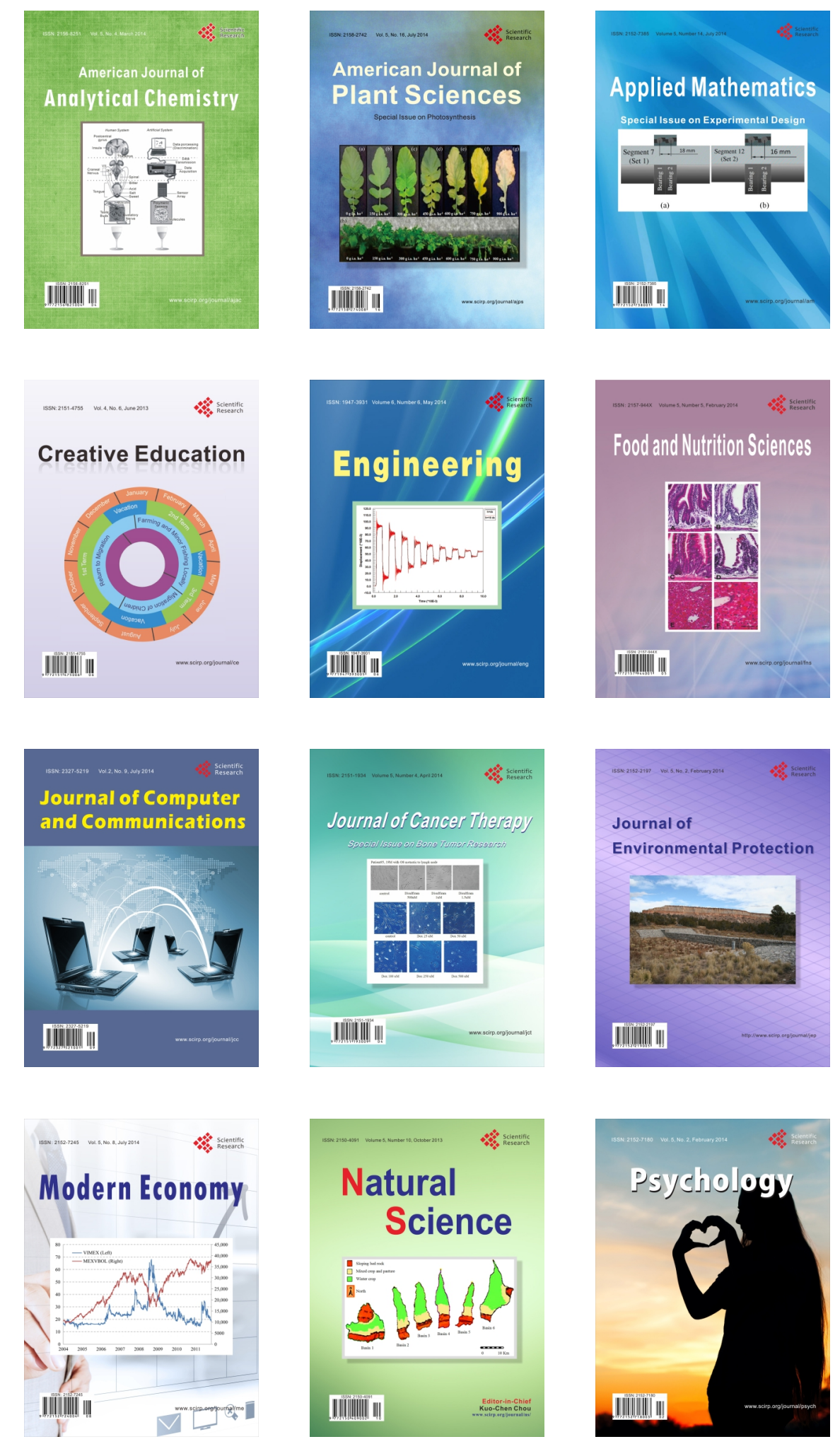